\title{
Treatment-resistant hypertension in a post-transplant patient with cystic fibrosis: a rare case of phaeochromocytoma
}

\section{David Joseph Tansey1', Jim John Egan2,5, Michelle Murray2,5, Katie Padfield3,5, John Conneely 4,5 and Mensud Hatunic ${ }^{1,5}$}

'Department of Endocrinology, 2Department of Respiratory Medicine and Lung \& Heart Transplantation, ${ }^{3}$ Department of Anesthesia, ${ }^{4}$ Department of General Surgery, Mater Misericordiae University Hospital, Dublin, Ireland, and 5UCD School of Medicine, University College Dublin, Belfield, Dublin, Ireland
Correspondence

should be addressed

to $\mathrm{M}$ Hatunic

Email

mhatunic@mater.ie

\section{Summary}

Phaeochromocytoma is a rare catecholamine-producing tumour. We present the case of phaeochromocytoma in a young man with a background history of a double-lung transplant for cystic fibrosis (CF). Clinical case: A 25-year-old man, with a background history of CF, CF-related diabetes (CFRD) and a double-lung transplant in 2012 was presented to the emergency department with crampy abdominal pain, nausea and vomiting. He was diagnosed with distal intestinal obstructions syndrome (DIOS). Contrast-enhanced CT imaging of the abdomen and pelvis showed a $3.4 \mathrm{~cm}$ right adrenal lesion. This was confirmed by a subsequent MRI of adrenal glands that demonstrated moderate FDG uptake, suggestive of a diagnosis of phaeochromocytoma. The patient was noted to be hypertensive with a blood pressure averaging 170/90 $\mathrm{mm} / \mathrm{Hg}$ despite treatment with three different anti-hypertensive medications - amlodipine, telmisartan and doxazosin. $\mathrm{He}$ had hypertension for the last 3 years and had noted increasingly frequent sweating episodes recently, without palpitations or headache. Laboratory analysis showed elevated plasma normetanephrines (NMN) of 3167 pmol/L (182-867) as well as elevated metanephrines (MN) of $793 \mathrm{pmol} / \mathrm{L}(61-377)$ and a high 3-MT of $257 \mathrm{pmol} / \mathrm{L}(<185)$. Once cathecholamine excess was identified biochemically, we proceeded to functional imaging to further investigate. MIBG scan showed a mild increase in the uptake of tracer to the right adrenal gland compared to the left. The case was discussed at a multidisciplinary (MDT) meeting at which the diagnosis of phaeochromocytoma was made. Following a challenging period of 4 weeks to control the patient's blood pressure with an alpha-blocker and beta-blocker, the patient had an elective right adrenalectomy, with normalisation of his blood pressure post-surgery. The histopathology of the excised adrenal gland was consistent with a 3 $\mathrm{cm}$ phaeochromocytoma with no adverse features associated with malignant potential.

\section{Learning points}

- Five to ten per cent of patients have a secondary cause for hypertension. Phaeochromocytomas are rare tumours, originating in chromaffin cells and they represent $0.1-1.0 \%$ of all secondary hypertension cases.

- Secondary causes should be investigated in cases where:

- Patient is presenting $<20$ years of age or $>50$ years of age,

- There is refractory hypertension, or

- There is serious end-organ damage present.

- Patients may present with the triad of headache, sweating and palpitations or more vague, non-specific symptoms. 
- Patients with suspected phaeochromocytoma should have 24-h urinary catecholamines measured and if available, plasma metanephrines measured. Those with abnormal biochemical tests should be further investigated with imaging to locate the tumour.

- Medical treatment involves alpha- and beta-blockade for at least 2 to 3 weeks before surgery as well as rehydration.

- There is a possibility of relapse so high-risk patients require life-long follow-up.

\section{Background}

Phaeochromocytoma is a rare tumour originating from catecholamine-secreting chromaffin cells that are derived from the ectodermic neural system and mostly situated within the adrenal medulla (1). This can result in catecholamine secretion in sudden bursts, leading to paroxysmal symptoms (2). It has an estimated incidence of less than $0.1 \%$ in the global population (3). However, their true incidence may be higher due to a lack of diagnosis until after death; a review of autopsy cases in Australia found that $0.05 \%$ had undiagnosed phaeochromocytoma (4).

The presentation of phaeochromocytomas vary from the classical symptom triad of palpitations, headaches and sweating to a spectrum of non-specific symptomatology, such as flushing, nausea, tiredness, weight loss or even no symptoms at all. All of these symptoms last from only minutes to hours and can occur periodically on different occasions (5). They are secondary to excess catecholamines. Phaeochromocytomas differ considerably in rates of catecholamine synthesis, turnover, and release, and in the types of catecholamines and metabolites produced. These differences may explain variations in presenting signs and symptoms; they also can provide useful information about the tumour, including the adrenal or extra-adrenal location, the underlying mutation, tumour size, and the presence of metastatic disease (6).

Regularly described as the 'great mimic' or 'great masquerader', the sometimes non-specific and often intermittent nature of the symptoms caused by phaeochromocytomas can result in its detection and diagnosis being missed by clinicians. Despite improved diagnostic techniques, there remains an approximate delay of 3 years between initial symptoms and the final diagnosis (7). This delay can lead to considerable morbidity and indeed mortality for patients.

We describe a case of an adrenal phaeochromocytoma in a young man with $\mathrm{CF}$, post lung transplant and multiple other co-morbidities. This case highlights the crucial importance of investigating secondary causes of hypertension.

\section{Case presentation}

A 25-year-old man, with a background history of CF, CFRD and a double-lung transplant in 2012 was presented to the emergency department with crampy abdominal pain, nausea and vomiting. He was diagnosed with DIOS, for which he was admitted for rehydration and laxatives. Contrast-enhanced CT imaging of the abdomen and pelvis showed a $3.4 \mathrm{~cm}$ right adrenal lesion, which was confirmed by a subsequent MRI of adrenals (Figs 1, 2A, B and $3 \mathrm{~A}, \mathrm{~B}, \mathrm{C})$.

His background medical history was notable for CF for which he underwent a double-lung transplant in 2012 and CFRD. He also had a history of gastrooesophageal reflux disease (GORD), for which he underwent a Nissen fundoplication in 2013, nasal polyps, previous episodes of DIOS, and he also had a low body mass density (BMD) for his age.

On review, the patient was noted to be hypertensive with a BP averaging 170/90 despite treatment with

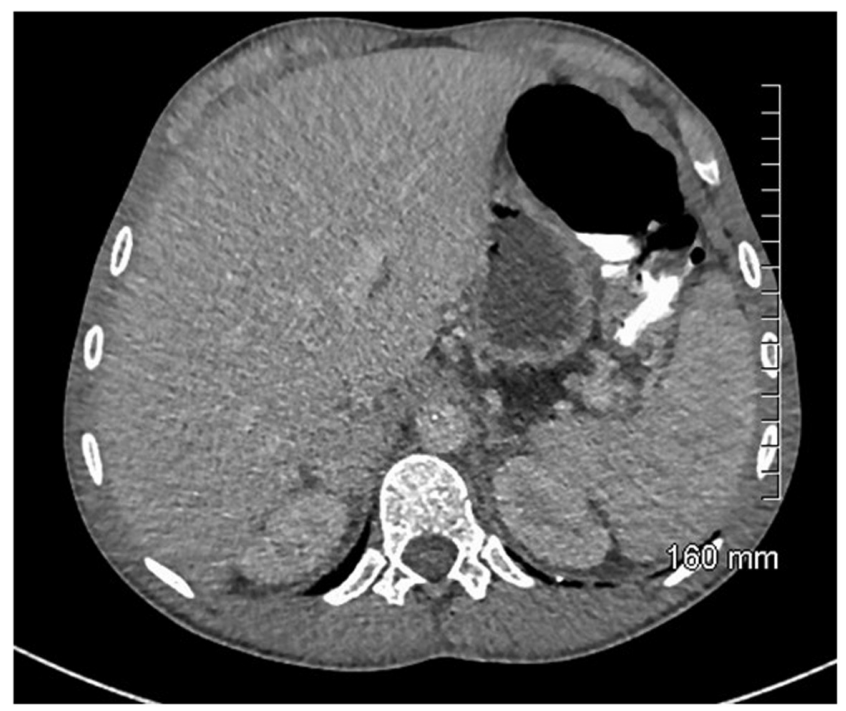

\section{Figure 1}

CT of thorax abdomen pelvis (TAP). CT of abdomen and pelvis 28/08/19 (portal venous phase) - right adrenal mass measuring $3 \times 2.5 \mathrm{~cm}$. Had been present since 2015 with minimal interval change in size. 

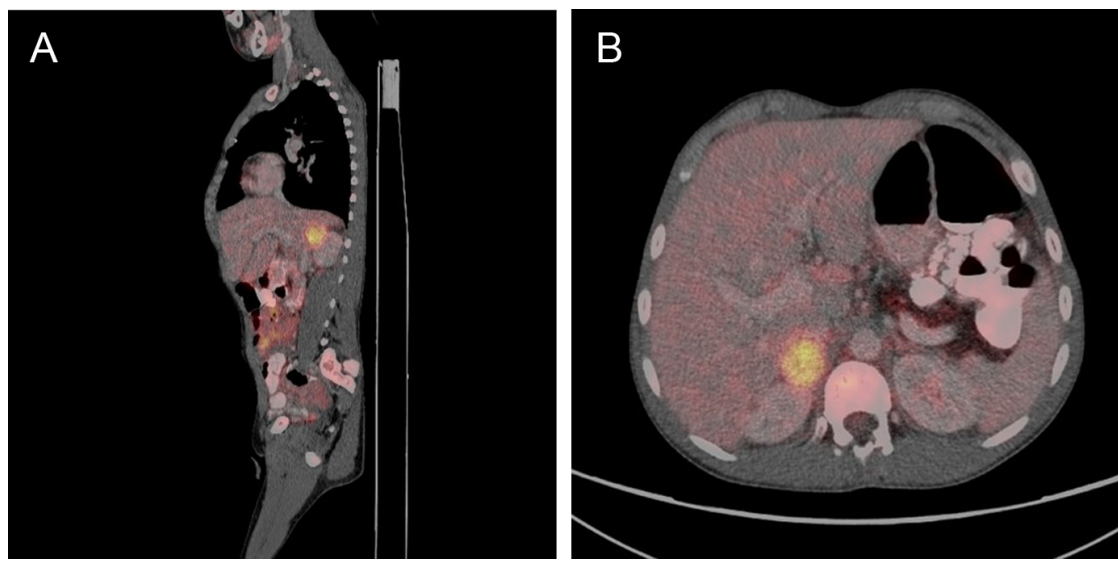

\section{Figure 2}

(A and B) PET CT. PET/CT 13/08/19 - carried out to assess the lymphadenopathy noted on MRCP. Sagittal view (left): anterior to the upper pole of the right kidney, there is an FDG-avid adrenal mass. Axial view (right).

maximum doses of three different anti-hypertensive medications - amlodipine, telmisartan and doxazosin. In the preceding 3 years, he had developed hypertension, in spite of significant increases in his anti-hypertensive medical therapy. He also complained of frequent sweating episodes over the past few months but denied any palpitations or headache.

\section{Investigation}

Biochemical: Our patient had a normal renal function, normal electrolytes, normal HbA1 and thyroid function. His liver function was abnormal with an alkaline phosphatase level of 1005 IU/L (30-130), ALT of 93 IU/I (0-55) and GGT of $684 \mathrm{IU} / \mathrm{L}(11-67)$. His albumin was normal at $36 \mathrm{~g} / \mathrm{L}$ (35-50), as was his bilirubin at $5 \mathrm{umol} / \mathrm{L}$ (5-24). Further laboratory analysis showed an elevated plasma normetanephrines (NMN) level of $3167 \mathrm{pmol} / \mathrm{L}$ (182-867) as well as elevated metanephrines (MN) level of $793 \mathrm{pmol} / \mathrm{L}$ (61-377) and high 3-MT of $257 \mathrm{pmol} / \mathrm{L}$ $(<185)$. His aldosterone was $<138 \mathrm{pmol} / \mathrm{L}$ (138-670), his renin was $173.1 \mathrm{mIU} / \mathrm{L}(9.0-103.5)$ and his AM cortisol was $69 \mathrm{nmol} / \mathrm{L}$ (150-455), in the setting of taking longterm steroids post-transplant in 2012.

Radiological: The CT of abdomen pelvis carried out to work up his GI disturbance on admission showed a right adrenal mass measuring $3 \times 2.5 \mathrm{~cm}$ that had been present since 2015 with minimal interval change in size (Fig. 1). This was confirmed by a subsequent MRI of adrenal glands. The PET CT showed that there was an FDG-avid adrenal mass, anterior to the upper pole of the right kidney (Fig. 2A). Finally, the MIBG scan showed only a mild increase in the uptake of tracer to the right adrenal gland compared to the left (Fig. 2B).

\section{Treatment}

Our patient's case was discussed at an MDT meeting at which, given the suggestive laboratory and radiologic findings, a diagnosis of phaeochromocytoma was made. The consensus decision was for an elective right adrenalectomy to excise the phaeochromocytoma.

The optimisation of blood pressure, as well as intravascular volume, is crucial to avoid perioperative adverse haemodynamic events, and we followed the preoperative guidelines and vitals targets recommended by the Endocrine Society Clinical Guidelines Subcommittee for patients undergoing PCC and PGL resection (8).

Controlling our patient's BP proved challenging. He was initially treated with phenoxybenzamine $10 \mathrm{mg}$ BD PO, however, it was required to up-titrate this to $10 \mathrm{mg}$ TDS PO then $20 \mathrm{mg}$ TDS PO over time due to persistent hypertension. We also started propranolol $10 \mathrm{mg}$ TDS also up-titrating it to $20 \mathrm{mg}$ TDS. Our target was for a BP of 110-100/50-70 with a heart rate of 50-70 bpm. The patient's underlying CF and DIOS may have contributed to malabsorption of these medications rendering preoperative control of his blood pressure more challenging.

The surgery had to be postponed on one occasion, due to inadequate BP control, further exacerbated by spikes in his BP caused by the patient's abdominal pain. He was treated with regular analgesia and his alphaand beta-blockade was further up-titrated. His eventual dose of phenoxybenzamine and propranolol was $70 \mathrm{mg}$ QDS and $40 \mathrm{mg}$ TDS, respectively. The peri-operative management included pre-hydration with IV $\mathrm{NaCl}$ at 100 $\mathrm{mL} / \mathrm{h}$ for $24 \mathrm{~h}$, increased oral salt intake, phentolamine IV as well as $100 \mathrm{mg}$ hydrocortisone IV at surgical induction. An elective laparoscopic right adrenalectomy and lymph node biopsy was then carried out. Post-surgery, our patient was treated with hydrocortisone $50 \mathrm{mg}$ IV TDS for 3 days 

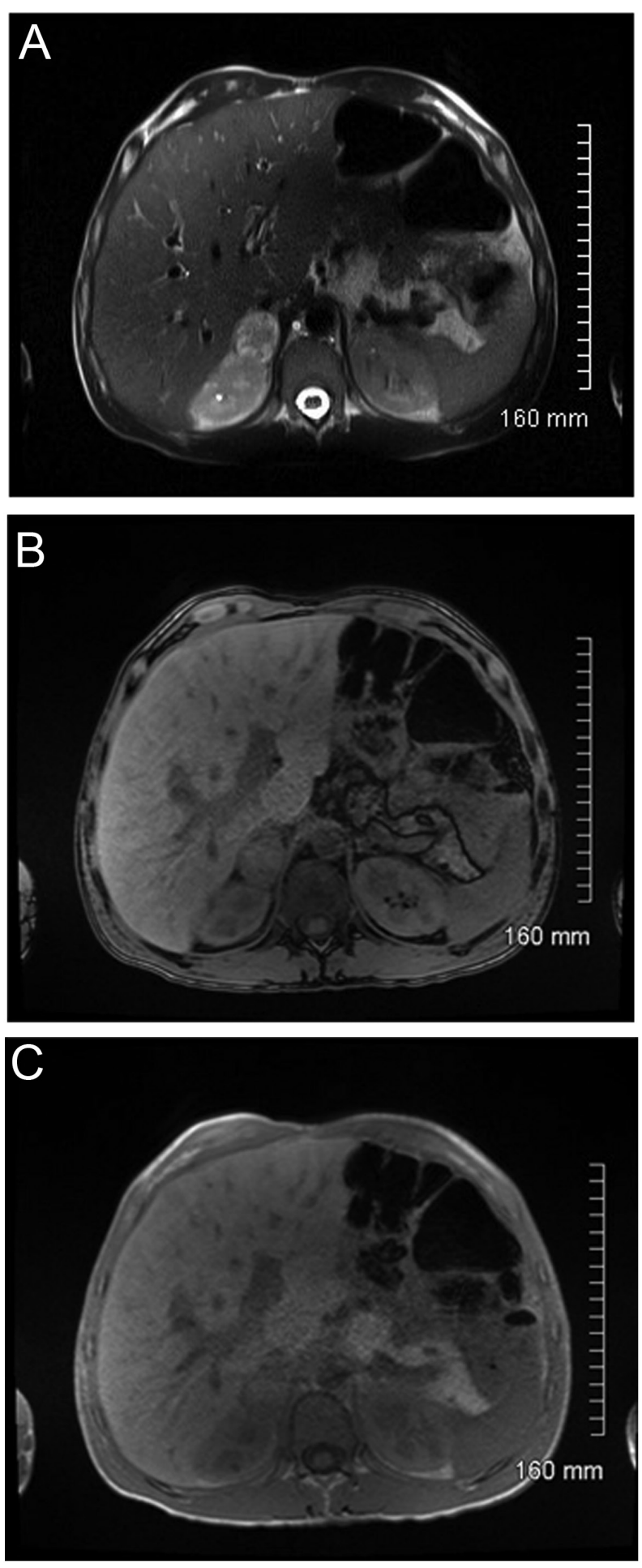

\section{Figure 3}

(A, B and C) MRI of the adrenal gland and MIBG: T2 haste sequence: heterogeneous mass in the right kidney. (B) out of phase (OOP). (C) In phase (IP). We compared the OOP to IP. If a lesion is full of fat and water (benign adenoma), it will be bright on IP but lose signal on OOP. This lesion stays the same on both OOP and IP. This can be seen in phaeochromocytoma. MIBG: mild increase in the uptake of tracer to the right adrenal gland compared to the left.
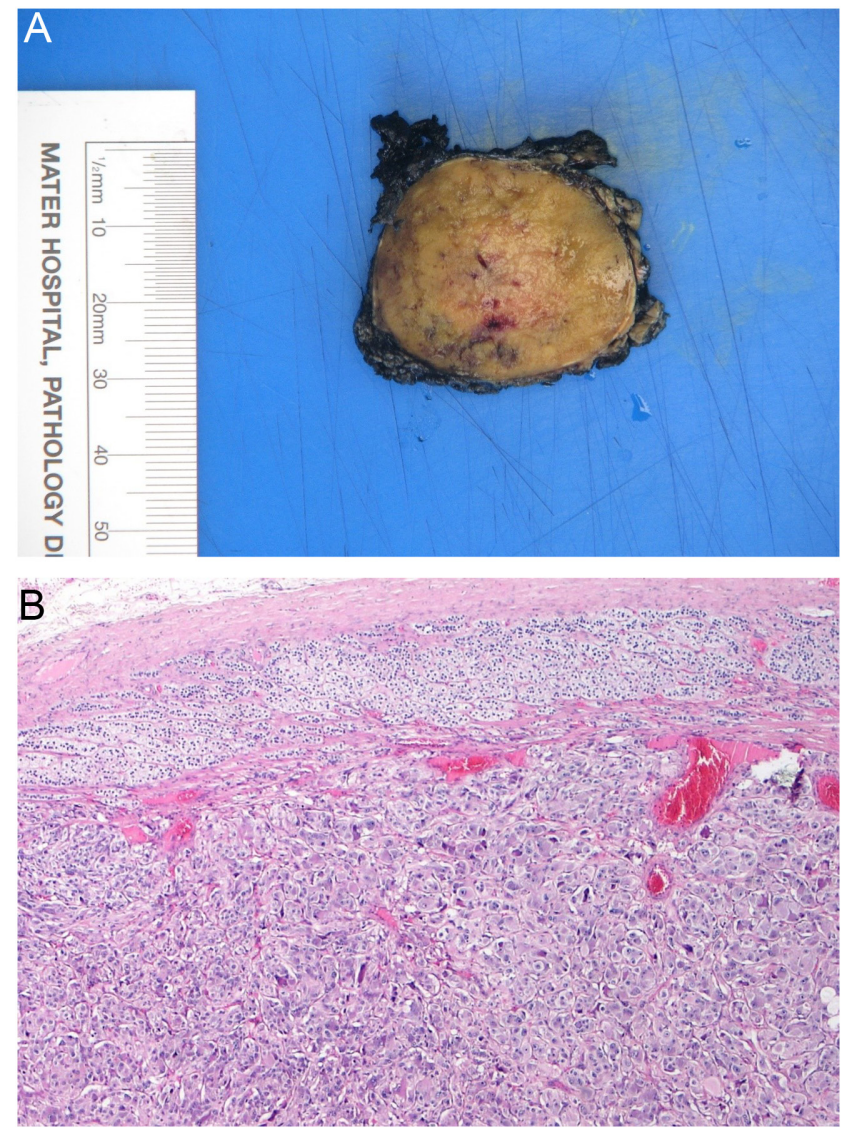

\section{Figure 4}

(A) Excised phaeochromocytoma on the right adrenal gland. The thin rim of adrenal cortex visible in areas. (B) Histological sample of excised pheochromoctyoma: circumscribed tumour occupying adrenal medulla with overlying normal adrenal cortical cells.

as well as a supplementary s.c. insulin sliding scale with a target glucose of $6-10 \mathrm{mmol} / \mathrm{mol}$. Our patient recovered well post-op, his blood pressure normalised completely, and he was discharged home 7 days post-surgery without any anti-hypertensive medication, with follow-up at the Endocrine and Transplant clinics.

\section{Outcome and follow-up}

Histology: The histopathology of the excised right adrenal gland showed a phaeochromocytoma, encapsulated tumour, $30 \mathrm{~mm}$ in maximum dimension, composed entirely of nests of large polygonal cells with abundant granular cytoplasm, large nucleoli and prominent nuclear pleomorphism. Vascularity was a prominent feature throughout, but there was no necrosis. No vascular invasion or extension into surrounding tissue was seen (Fig. 4A and B). The PASS score showed a low risk for aggressive behaviour or malignancy with a value of $2 / 20$ 
$(<4 / 20)$. The mesenteric lymph nodes showed no evidence of lymphoma or lymphoproliferative disorder.

The patient returned to our Endocrine outpatient clinic in December 'feeling great'. His blood pressure had settled to $123 / 82 \mathrm{~mm} / \mathrm{Hg}$ without any anti-hypertensive medication, and he denied any further episodes of sweating/flushing since the surgery. Repetition of biochemical analysis showed normal metanephrines of 72 $\mathrm{pmol} / \mathrm{L}$ (61-377), normal normetanephrines of $94 \mathrm{pmol} / \mathrm{L}$ (182-867) and normal 3-MT of $<65$ pmol/L $(<185)$.

\section{Discussion}

Our case of a young male patient with a history of hypertension who was presented with abdominal pain highlights the importance of screening for secondary aetiologies of hypertension in this population. The differential diagnosis includes renal artery stenosis caused by fibromuscular dysplasia, which is one of the most common secondary aetiologies, particularly in younger patients. Atherosclerotic renal artery stenosis is also a major cause of secondary hypertension in older adults. In middle-aged adults, aldosteronism is the most common secondary cause of hypertension, and the recommended initial diagnostic test is an aldosterone/renin ratio (9). Other differentials include Cushing's syndrome, thyroid dysfunction and obstructive sleep apnoea (10).

Our patient had a history of treatment-resistant hypertension for 3 years, despite being on 3 antihypertensive agents. His high blood pressure on admission was attributed to be secondary to the abdominal pain that he was experiencing. However, during the work-up, an abdominal CT showed a mass in the right adrenal gland. Based on these findings, phaeochromocytoma was suspected, which was subsequently confirmed by ancillary tests and pathology. Nausea and abdominal pain are recognised gastrointestinal symptoms of a phaeochromocytoma. Mullen et al. found that chronic constipation was noted in $13 \%$ of patients with phaeochromocytoma (11). Interestingly, our patient had multiple admissions to hospital for constipation and was diagnosed with DIOS, an issue which may have been exacerbated by both his cystic fibrosis and the catecholamine excess caused by the phaeochromocytoma. Our patient had a high 3-MT level, most commonly associated with extra-adrenal tumours, genetic mutations or malignant tumours. For this reason, we have sent our patient for genetic testing and continue his follow-up for life.
Phaeochromocytomas have been associated with a variety of cardiovascular complications due to overwhelming catecholamine levels. The most common complications include left ventricular hypertrophy, ischaemic heart disease, disturbances in rhythm and conduction, malignant ventricular arrhythmias, and even shock (12). The potentially fatal cardiovascular complications of these tumours are due to the potent effects of catecholamines, especially noradrenaline, the main transmitter released from sympathetic nerve terminals. Therefore, the longer that a diagnosis of phaeochromocytoma is missed, the greater the potential morbidity and mortality for these patients.

We describe a rare case of a right adrenal phaeochromocytoma in a young man with multiple co-morbidities, who completely recovered after tumour resection. This case highlights the crucial importance of investigating secondary causes of hypertension, even in patients with multiple comorbidities. The diagnosis of phaeochromocytoma requires high clinical alertness due to its rare incidence and extremely variable clinical presentation. It also highlights the relevance of considering phaeochromocytoma in the differential diagnosis of unexplained hypertension.

\section{Declaration of interest}

The authors declare that there is no conflict of interest that could be perceived as prejudicing the impartiality of the research reported.

\section{Funding}

This research did not receive any specific grant from any funding agency in the public, commercial or not-for-profit sector.

\section{Patient consent}

The author confirms that written informed consent has been obtained from the patient for publication of the submitted article and accompanying images.

Author contribution statement

All authors listed contributed equally to this paper and were the primary physicians involved in the care of the patient.

\section{References}

1 Neumann HP. Pheocromocytoma. Harrisons Principles of Internal Medicine, 17th ed. New York: Mcgraw-Hill Medical, 2008.

2 Därr R, Lenders JW, Hofbauer LC, Naumann B, Bornstein SR \& Eisenhofer G. Pheochromocytoma - update on disease management. Therapeutic Advances in Endocrinology and Metabolism 20123 11-26. (https://doi.org/10.1177/2042018812437356) 
3 Farrugia FA, Marikos G, Tzanetis P, Charalampopoulos A, Misiakos E, Zavras N \& Sotiropoulos D. Pheochromocytoma, diagnosis and treatment: review of the literature. Endocrine Regulations $2017 \mathbf{5 1}$ 168-181. (https://doi.org/10.1515/enr-2017-0018)

4 McNeil AR, Blok BH, Koelmeyer TD, Burke MP \& Hilton JM.

Phaeochromocytomas discovered during coronial autopsies in Sydney, Melbourne and Auckland. Australian and New Zealand Journal of Medicine 200030 648-652. (https://doi.org/10.1111/j.1445-5994.2000.tb04358.x)

5 Manger WM \& Gifford RW. Clinical and Experimental Pheochromocytoma. Blackwell Science, 1996.

6 Pacak K. Preoperative management of the pheochromocytoma patient. Journal of Clinical Endocrinology and Metabolism 200792 4069-4079. (https://doi.org/10.1210/jc.2007-1720)

7 Lenders JW, Eisenhofer G, Mannelli M \& Pacak K. Phaeochromocytoma. Lancet 2005366 665-675. (https://doi. org/10.1016/S0140-6736(05)67139-5)

8 Lenders JW, Duh QY, Eisenhofer G, Gimenez-Roqueplo AP, Grebe SK, Murad MH, Naruse M, Pacak K, Young Jr WF \& Endocrine Society. Pheochromocytoma and paraganglioma: an endocrine society clinical practice guideline. Journal of Clinical Endocrinology and Metabolism 201499 1915-1942. (https://doi.org/10.1210/jc.20141498)

9 Viera AJ \& Neutze DM. Diagnosis of secondary hypertension: an age-based approach. American Family Physician 201082 1471-1478.

10 Adas M, Koc B, Adas G, Yalcin O, Celik S \& Kemik Ö. Pitfalls in the diagnosis of pheochromocytomas: a case series and review of the literature. Journal of Epidemiological Research 20162 49-55. (https:// doi.org/10.5430/jer.v2n2p49)

11 Mullen JP, Cartwright RC, Tisherman SE, Misage JR \& Shapiro AP. Pathogenesis and pharmacologic management of pseudo-obstruction of the bowel in pheochromocytoma. American Journal of the Medical Sciences 1985290 155-158. (https://doi.org/10.1097/00000441198510000-00004)

12 Galetta F, Franzoni F, Bernini G, Poupak F, Carpi A, Cini G, Tocchini L, Antonelli A \& Santoro G. Cardiovascular complications in patients with pheochromocytoma: a mini-review. Biomedicine and Pharmacotherapy 201064 505-509. (https://doi.org/10.1016/j. biopha.2009.09.014)

Received in final form 23 April 2021

Accepted 27 April 2021 